\section{International Scientific Journal Theoretical \& Applied Science}

p-ISSN: 2308-4944 (print) e-ISSN: 2409-0085 (online)

Year: $2016 \quad$ Issue: $4 \quad$ Volume: 36

Published: $30.04 .2016 \quad$ http://T-Science.org

SECTION 1. Theoretical research in mathematics.
Nariman Kiyalbekov teacher of the highest of categories, teacher regional school boarding school of "Daryn", Taraz nariman_kiyalbekov@mail.ru

Razila Beshtayeva master of mathematics, teacher TarSU of M.Kh.Dulati razila-83@mail.ru

Karligash Taubayeva student of the 2 nd course of TARGU

Boomgirl_1996@mail.ru

Diana Mekemova student of the 2 nd course of TARGU diko_96_45@mail.ru

\title{
MODELLING NEGATIVE IMPACT OF NONFREEZING RESERVOIRS ON THE ENVIRONMENT MICROCLIMATE
}

\author{
Abstract: For research of anthropogenous influence on a mesolarge-scale microclimate with participation of \\ the person the three-dimensional mathematical model, time-dependent is offered. Influence of the fog arising near \\ not freezing pools on a microclimate is considered \\ Key words: nonfreezing reservoir, thermal capacity, density, kinetic turbulent energy, fogging. \\ Language: Russian \\ Citation: Kiyalbekov N, Beshtayeva R, Taubayeva K, Mekemova D (2016) MODELLING NEGATIVE \\ IMPACT OF NONFREEZING RESERVOIRS ON THE ENVIRONMENT MICROCLIMATE. ISJ Theoretical \& \\ Applied Science, 04 (36): 205-210. \\ Soi: http://s-o-i.org/1.1/TAS-04-36-36 Doi: crossef http://dx.doi.org/10.15863/TAS.2016.04.36.36 \\ УДК 551.46: 628.5

\section{МОДЕЛИРОВАНИЕ НЕГАТИВНОГО ВОЗДЕЙСТВИЯ НЕЗАМЕРЗАЮЩИХ ВОДОЕМОВ НА МИКРОКЛИМАТ ОКРУЖАЮЩЕЙ СРЕДЫ}

Аннотация: Для исследования антропогенного влияния на мезомасштабный микроклимат с участием человека предлагается трехмерная математическая модель, зависящая от времени. Рассматривается влияние тумана, возникаюшего возле не замерзающих бассейнов, на микроклимат

Ключевые слова: незамерзающий водоем, теплоемкость, плотность, кинетическая турбулентная энергия, туманообразования.

В последние годы в связи с растущими энергетическими потребностями народного хозяйства возросла необходимость строительства электростанций в различных районах страны. Следствием этого является создание многочисленных прудов-охладителей и водохранилищ. Появления новых водоемов может вызывать изменение в микроклимате прилегающих районов и тем самым повлиять на условия жизни населения, на сроки сельскохозяиственных работ и т. д. Аналогичные возмущении микроклимата и туманы, наблюдаемые в природе, - это «речные и морские» туманы парения [1]. Они обычно образуются в зимний и осенне - весенний сезоны при адвекции холодного воздуха на более теплой водной подстилающей поверхности. Это может быть естественный водоем: река, озеро, море, - но особенно часто искусственный: незамерзающее водохранилище, верхний и особенно нижний бьефе ГЭС, где незамерзающие полыньи с туманами, образующиеся после строительства плотин, могут простираться на 100-150км. Даже если туман не образуется, то с подветренной стороны от водоема наблюдается заметные повышения температуры и влажности, гололедно - изморозевные отложения существенно ухудщающие микроклимат, также, как и влизи АЭС и ТЭС.

Для уменьшения негативного влияния туманов парения необходимо прежде всего знать характеристики туманов: протяженность, 


\begin{tabular}{|c|c|c|c|c|c|c|}
\hline Impact Factor: & $\begin{array}{l}\text { ISRA (India) } \\
\text { ISI (Dubai, UAB } \\
\text { GIF (Australia) } \\
\text { JIF }\end{array}$ & $\begin{array}{r}=1.344 \\
=0.829 \\
=0.564 \\
=1.500\end{array}$ & $\begin{array}{l}\text { SIS (USA) } \\
\text { PИHЦ (Russia) } \\
\text { ESJI (KZ) } \\
\text { SJIF (Morocco) }\end{array}$ & $\begin{array}{l}=0.912 \\
=0.234 \\
=1.042 \\
=2.031\end{array}$ & $\begin{array}{l}\text { ICV (Poland) } \\
\text { PIF (India) } \\
\text { IBI (India) }\end{array}$ & $\begin{array}{l}=6.630 \\
=1.940 \\
=4.260\end{array}$ \\
\hline
\end{tabular}

микроструктуру, дальность видимости и зависимость этих характеристик от параметров водоема и метеорологических условии. В холодное время года в окрестности этих водоемов резко возрастает потенциальная возможность образования переохлажденных туманов и, соответственно, - возможность возникновения одного из опасных метеорологических явлении - гололеда. Обледенению подвергаются высотные сооружения, линии связи и линии электропередач.

В предлагаемой математической модели рассчитываются динамика атмосферного пограничного слоя включая процессы турбулентной диффузии, эволюция полей температуры и влажности воздуха; кинетика фазовых переходов влаги, имеющая большое значения при изучении туманообразования кроме того рассчитываются скорости радиационного выхоложивания, нагрева радиационной баланс поверхности, определяющий суточный ход температуры. \begin{tabular}{cccc}
\multicolumn{2}{c}{ Уравнения } & модели & дополняются \\
начальными & и & краевыми & условиями,
\end{tabular} описывающими конкретный физический процесс. В данной работе исследуется возможное воздействие незамерзающих водоемов на атмосферу. Особое внимание было уделено условиям возможного туманообразования в результате взаимодействия холодных воздушных масс в пограничном слое с теплой поверхностью водоема.

В модели рассчитывается динамика пограничного слоя неразрывности для горизонтальных $u, v$ и вертикального $w$ компонентов вектора скорости: уравнения баланса турбулентной энергии в, соотношениям подобия и размерности для пути смещения $l$, вертикального коэффициента турбулентности $K_{Z}$ и скорости диссипации турбулентной энергии $\boldsymbol{E}$, дополненной гипотезой предложенный $[1,2,3]$ :

$$
\begin{aligned}
& \frac{\partial u}{\partial t}+(\vec{u}, \nabla u)=-\frac{\partial \pi^{\prime}}{\partial x}+\frac{\partial}{\partial z} K_{Z} \frac{\partial u}{\partial z}+K_{M} \Delta u+f_{k}(v-V) \\
& \frac{\partial v}{\partial t}+(\vec{u}, \nabla v)=-\frac{\partial \pi^{\prime}}{\partial y}+\frac{\partial}{\partial z} K_{Z} \frac{\partial v}{\partial z}+K_{M} \Delta v-f_{k}(u-U) \\
& \frac{\partial w}{\partial t}+(\vec{u}, \nabla w)=-\frac{\partial \pi^{\prime}}{\partial z}-\lambda \vartheta^{\prime}+\frac{\partial}{\partial z} K_{Z} \frac{\partial w}{\partial z}+K_{M} \Delta w \\
& \frac{\partial u}{\partial x}+\frac{\partial v}{\partial y}+\frac{\partial w}{\partial x}=0
\end{aligned}
$$

Поля температуры и влажности рассчитывались из следующих уравнений:

$$
\begin{gathered}
\frac{\partial \vartheta^{\prime}}{\partial t}+\left(\vec{u}, \nabla \vartheta^{\prime}\right)+S w=\frac{\partial}{\partial z} K_{Z} \frac{\partial \vartheta^{\prime}}{\partial z}+\Delta \vartheta^{\prime}+\frac{L_{1}}{C_{P}} \Phi_{1}+\frac{L_{1}}{C_{P}} \Phi_{2}+R_{r a d} \\
\frac{\partial q^{\prime}}{\partial t}+\left(\vec{u}, \nabla q^{\prime}\right)+\gamma_{q} w=\frac{\partial}{\partial z} K_{Z} \frac{\partial q^{\prime}}{\partial z}+\Delta q^{\prime}-\Phi
\end{gathered}
$$

Для замыкания системы уравнения (1) - (5) относительно вертикального коэффициента турбулентного обмена для тепла $K_{H}$ и импульса

$$
\begin{aligned}
& \frac{\partial b^{2}}{\partial t}+\bar{u} g r a d b^{2}=\frac{\partial}{\partial z^{*}}\left[E_{3} b l \frac{\partial b^{2}}{\partial z^{*}}\right]+2\left[-\overline{u w} \frac{\partial u}{\partial z^{*}}-\overline{v w} \frac{\partial v}{\partial z}\right]+ \\
& +2 \beta g \overline{w \bar{\theta}}-2 \frac{b^{3}}{B_{1} l},
\end{aligned}
$$

движения $K_{M}$ использовались кинетические уравнения турбулентной энергии $[1,3,4]$ : где $\bar{\theta}$ - средняя по вертикали фоновая потенциальная температура;

$\beta$ - коэффициент объемного теплового расширения;
Турбулентные потоки определим с помощью градиентный аппроксимации: 


\begin{tabular}{|c|c|c|c|c|c|c|}
\hline Impact Factor: & $\begin{array}{l}\text { ISRA (India) } \\
\text { ISI (Dubai, UAE } \\
\text { GIF (Australia) } \\
\text { JIF }\end{array}$ & $\begin{array}{l}=1.344 \\
=0.829 \\
=0.564 \\
=1.500\end{array}$ & $\begin{array}{l}\text { SIS (USA) } \\
\text { PИНЦ (Russia) } \\
\text { ESJI (KZ) } \\
\text { SJIF (Morocco) }\end{array}$ & $\begin{array}{l}=0.912 \\
=0.234 \\
=1.042 \\
=2.031\end{array}$ & $\begin{array}{l}\text { ICV (Poland) } \\
\text { PIF (India) } \\
\text { IBI (India) }\end{array}$ & $\begin{array}{l}=6.630 \\
=1.940 \\
=4.260\end{array}$ \\
\hline
\end{tabular}

$(-\overline{u w},-\overline{v w})=a_{3} K_{M}\left(\frac{\partial u}{\partial z^{*}}, \frac{\partial v}{\partial x^{*}}\right)$

$K_{M} \quad$ и $\quad$ тепла $K_{H}$ определяются из $-\overline{w \theta}=a_{3} K_{H} \frac{\partial \vartheta}{\partial z^{*}}$. алгебраических выражений, а путь смещения $l$ в зависимости от класса устойчивости в слоях атмосферы:

В данной работе значения коэффициентов турбулентного обмена для импульса движения

$$
\begin{gathered}
K_{H}=A_{1} l b^{3}\left[(1-3 C) b^{2}+3 A_{2} l^{2}\left\{\left(B_{2}-3 A_{2}\right)-3 C\left(4 A_{1}+B_{2}\right)\right\} S\right]+ \\
{\left[\begin{array}{l}
b^{4}+6 A_{1}^{2} l^{2} b^{2}\left|\frac{\partial \bar{v}_{z}^{2}}{\partial z}\right|^{2}+ \\
+3 A_{1} A_{2} l^{2}\left\{6 A_{1}^{2} l^{2}\left(B_{2}-3 A_{2}\right)\left|\frac{\partial \bar{v}_{z}}{\partial z}\right|^{2}+\left(7+B_{2} / A_{2}\right) b^{2}+9 A_{2} l^{2}\left(4 A_{1}+B_{2}\right) S\right\} S
\end{array}\right]} \\
K_{H}=A_{2} l\left[b^{3}-6 A_{1} l\left|\frac{\partial \bar{v}_{z}}{\partial z}\right|^{2}\right]+\left[b^{2}+3 A_{2} l^{2}\left(4 A_{1}+B_{2}\right) \cdot S\right]
\end{gathered}
$$

Здесь,

$$
b^{2}=\left(u^{\prime 2}+v^{\prime 2}+w^{\prime 2}\right) / 2
$$

значения

кинетическая турбулентная энергия; $l$ - масштаб турбулентности (путь смещения), $\mathrm{S}$ - параметр стратификации; ( $\left.\mathrm{A}_{1}, \mathrm{~A}_{2}, \mathrm{~B}_{1}, \mathrm{~B}_{2} \mathrm{C}, \mathrm{E}_{3}\right)$ - эмпирическая постоянные. В расчетах принимаются следующие

$$
\left(\mathrm{A}_{1}, \mathrm{~A}_{2}, \mathrm{~B}_{1}, \mathrm{~B}_{2}, \mathrm{C}, \mathrm{E}_{3}\right)=(1.031,0.4532,42.5,15.1,0.2,1) .
$$

Масштаб турбулентности $l$ определяется в ПСА следующим образом:

- для конвективного слоя атмосферы:

$$
l=\frac{\chi}{B_{1}}\left\{\frac{2\left(12+0,5 \frac{z_{i}}{|L|}\right)^{2 / 3}+1,7\left(1+3 z_{i} \times|L|\right)^{2 / 3}}{1+0,5|z \times L|^{2 / 3}}\right\}
$$

- для слабоустойчивого слоя:

$$
l=\left\{\frac{\left(0,62+1,8\left(\frac{z}{z_{i}}\right)^{2 / 3}\right)-1,65\left(\frac{z}{z_{i}}\right)^{4 / 3}}{B_{1}\left(0,6\left(\frac{z}{z_{i}}\right)^{-1 / 3}-0,3\right)}\right\}
$$

- для устойчивого и нейтрального слоя атмосферы примем:

$$
\begin{gathered}
\frac{1}{l}=\frac{\Phi_{l}}{\chi}+\frac{1}{l_{0}}, \Phi_{l}=1+3,3 \frac{z}{l}, \\
l_{0}=0,1\left(\int_{0}^{\infty} b z d z / \int_{0}^{\infty} b d z\right),
\end{gathered}
$$

где $\chi$ - постоянная Карамана; $z_{i}$ - высота конвективного слоя;

$L=-u_{*}^{3} / \chi^{3} \lambda \vartheta$ - масштаб длины МониноОбухова.
Здесь $\vec{u}$ - трехмерной вектор скорости, $\Delta=\partial^{2} / \partial x^{2}+\partial^{2} / \partial y^{2}$ оператор Лапласа; $f_{k}, \lambda$ - параметры Кориолиса и конвекции; $V_{g}(z), U_{g}(z)$ - компоненты геострофического ветра с учетом термического ветра (бароклинности); $\vartheta^{\prime}$ - отклонения потенциальной температуры с фоновой; $L_{1}, L_{2}$ - скрытая удельная теплота конденсации и сублимации; $C_{p}$ - удельная теплоемкость воздуха при постоянном 


\begin{tabular}{|c|c|c|c|c|c|c|}
\hline Impact Factor: & $\begin{array}{l}\text { ISRA (India) } \\
\text { ISI (Dubai, UAF } \\
\text { GIF (Australia) } \\
\text { JIF }\end{array}$ & $\begin{array}{l}=1.344 \\
=0.829 \\
=0.564 \\
=1.500\end{array}$ & $\begin{array}{l}\text { SIS (USA) } \\
\text { PИНЦ (Russia) } \\
\text { ESJI (KZ) } \\
\text { SJIF (Morocco) }\end{array}$ & $\begin{array}{l}=0.912 \\
=0.234 \\
=1.042 \\
=\mathbf{2 . 0 3 1}\end{array}$ & $\begin{array}{l}\text { ICV (Poland) } \\
\text { PIF (India) } \\
\text { IBI (India) }\end{array}$ & $\begin{array}{l}=6.630 \\
=1.940 \\
=4.260\end{array}$ \\
\hline
\end{tabular}

давлении; $R_{\text {rad }}$ - скорость радиационного выхолаживания; q- удельная влажность; $\Phi_{1}, \Phi_{2}$ -скорости конденсации и сублимации;

Для расчета эволюции микрофизических процессов в районе незамерзающих водоемов необходимо решать уравнения для функций распределения капель $f_{1}\left(\vec{x}, r_{1}, t\right)$ и кристаллов $f_{2}\left(\vec{x}, r_{2}, t\right)$ совместно с уравнениями (1) - (2).

Для расчета скорости радиационного выхолаживания в слое тумана и эффективного излучения поверхности $R_{0}$ решались уравнения переноса длинноволновой радиации в двух потоковом приближении. Использовался метод схематизированного спектра К.Я. Кондратьева.

Краевые условия на подстилающей поверхности (на уровне шероховатостей $z_{0}$ ) вокруг незамерзающей водоема следующие: - для температуры - уравнения теплового баланса $[1,3,6]$ :

$$
-\rho_{a} c_{p} k_{0}\left(\frac{\partial T}{\partial z}+\gamma_{a}\right)-L_{w} \rho_{a} k_{0} \frac{\partial q}{\partial z}-c_{S} \rho_{S} k_{S} \frac{\partial T_{S}}{\partial z}=F_{l}^{\uparrow}-F_{l}^{\downarrow},
$$

которые решалось вместе с уравнением теплопроводности в почве

$$
\frac{\partial T_{S}}{\partial t}=\frac{\partial}{\partial z} k_{S} \frac{\partial T_{S}}{\partial z}+\eta(z, t)
$$

- для влажности - условия сшивки молекулярного турбулентного потоков пара

$$
-k_{0} \rho_{a} \frac{\partial q}{\partial z}=\alpha_{э \phi} \rho_{a} \frac{V_{\Pi}}{4}\left(q_{S 1}-q_{0}\right) .
$$

Здесь $\gamma_{a}$ - сухоадиабатический градиент температуры; $\alpha_{\text {эф }}$ - эффективный коэффициент конденсации на поверхности; $c_{S}, \rho_{S}, k_{S}, T_{S}$ теплоемкость, плотность, температурапроводность и температура почвы соответственно. $F_{l}^{\uparrow^{\downarrow}}$ - интегральные потоки радиации. При расчете длинноволнового излучения используется традиционный подход с интегральными функциями пропускания, которые являются функцией температуры и концентрации основных поглотителей: водяного пара, жидкокапельной воды и углекислого газа. При расчете интегральных функций пропускания используются аппроксимации, предложенные в

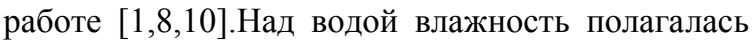
равной насыщающей, температура воды - $0^{0} \mathrm{C}$ а влажность над поверхностью варьировалась в различных экспериментах; кроме того, на подстилающей поверхности.

Для решения дифференциальные уравнения (1) - (5) с соответствующими краевыми условиями по времени применяется метод расщепления по физическим фактором [3]. Конечно - разностные аппроксимации получаются из дискретизации сумматорного тождества. На каждом временном интервале будем расщеплять задачу на два этапа:

1. Перенос субстанции вдоль траекторий и турбулентной обмен,
2. Задачи динамического метеорологических полей

Первый этап соответсвует с начальными и краевыми условиями с помощью покомпонентого расщепления [3] сводится к последовательному решению ряда одномерных задач. На втором этапе решается задача динамического согласования метеорологических полей, алгоритм которой подробно описан в работах $[1,3]$. Уравнения турбулентной энергии решалось методом простой прогонки с применением итерационной процедуры.

С помощью описанной модели была проведена серия численных расчетов по моделированию мезометеорологических процессов под влиянием водохранилища. Линейные размеры незамерзающего водоема $6 \mathrm{KM}^{2}$, а рассматриваемый полигон по $\mathrm{X}$ и $\mathrm{Y}$ составил 60км ${ }^{2}$ и по вертикали 2,5км. Эти размеры были выбраны исходя из условия, что они больше возможных максимальных масштабов локальных атмосферных процессов. Расчетная сетка имела 30 узлов по вертикали и 30 узлов по горизонтали. По вертикали сетка была неравномерной ее шаги по оси $\mathrm{Z}$ возрастали от $5 \mathrm{M}$ из подстилающей поверхности до $100 \mathrm{M}$ верхней части области. Остальные параметры модели были выбраны следующие:

$\mathrm{f}_{\mathrm{k}} \quad=10^{-4} \mathrm{c}^{-1}, \quad \mathrm{k}_{\mathrm{M}} \quad=10^{4} \mathrm{M}^{2} / \mathrm{c}, \quad \mathrm{S}=0,45 \cdot 10^{-2} г$ рад $/ \mathrm{M}$, $\lambda=0,046 \mathrm{M} /(\mathrm{c} \cdot$ град),

$\mathrm{Z}_{0}=0,01 \mathrm{M}$;

$$
=0,4 \text {, }
$$

$$
\rho_{a}=1300 \Gamma / \mathrm{M}^{3}
$$

$\mathrm{c}_{\mathrm{p}}=0,24$ кал/(г·град);

$\rho_{S} c_{S}=44 \cdot 10^{4}$ кал/( $\left.{ }^{3} \cdot г р а д\right)$;

$\mathrm{k}_{\mathrm{S}}=3 \cdot 10 \mathrm{M}^{2} / \mathrm{c}$, $\mathrm{g}=9,8 \mathrm{M} / \mathrm{c}^{2}, \mathrm{a}_{3}=0,56, \mathrm{~B}_{3}=0,08$.

$\theta=\bar{\theta}+S z_{1}, \bar{\theta}=290 \mathrm{k}$.

Температуры водоема окружающего воздуха и их разница задавались характерными для осеннего времени. Варианты расчетов приведена в таблице 1 . 
Рассмотрим вариант эксперимента, в котором задавалась средняя температура водоема $+10^{\circ} \mathrm{C}$. Начальная температура воздуха $-10^{\circ} \mathrm{C}$, затем через 2,5 - 3 года она понижается на $3-4^{0} \mathrm{C}$ вследствие радиационного выхоложивание, т.е. горизонтальный контраст температуры $\Delta T=22$ $23^{\circ} \mathrm{C}$.

Вопрос инициализации принадлежат к числу наименее разработанных в задаче мезомасштабного численного прогноза. В связи с отсутствием мезомасштабной сети наблюдений предпологают обычно, что указанная задача является задачей "без начальных данных", другими словами, что влияние начальных полей становится пренебрежимым уже в первые часы прогноза, и в дальнейшем прогноз определяется вынужденными воздействиями в сочетании со свойствами данного региона (радиационные притоки тепла, вторжение извне, орография, альбедо и т.д.). Поэтому начальные поля обычно восстанавливается по тем или иным стационарным квазистатическим соотношением с целью сократить начальный период их резкой перестройки.

Таблица 1

\section{Варианты расчетов естественной эволюции тумана.}

\begin{tabular}{|c|c|c|c|c|c|c|}
\hline Вариант & $\vec{u}_{\Phi^{\mathrm{M} / \mathrm{c}}}$ & $\mathrm{q}_{0} \%$ & $\mathrm{~T}_{1}=\mathrm{T}_{2}{ }^{0} \mathrm{C}$ & $\begin{array}{c}\max \text { водность } \\
\text { Г/кГ }\end{array}$ & время развития мик & $\begin{array}{c}\text { ледность } \\
\Gamma / \mathrm{M}^{3}\end{array}$ \\
\hline 1 & 5 & 90 & -10 & 0,29 & 52 & - \\
\hline 2 & 5 & 85 & -10 & 0,21 & 59 & - \\
\hline 3 & 3 & 85 & -10 & 0,24 & 48 & - \\
\hline 4 & 3 & 80 & -10 & 0,25 & 55 & - \\
\hline 5 & 5 & 92 & -12 & 0,42 & 37 & 0,005 \\
\hline
\end{tabular}

Влажность над водоемом 3- 4 раза больше чем окружающей суши, возмущения температуры максимальны на высоте $120-150$ м, а на подстилающей поверхности быстро релаксируют. Результаты расчетов приводится на момент времени через 1ч после момента начала интегрирования. К этому времени характерные параметры атмосферы выходят на квазистационарный уровень определяемый скоростью длиноволнового радиационного выхолаживания. В результате взаимодействия холодной воздушной массы $\mathrm{T}_{1}=-10^{\circ} \mathrm{C}$ в набегающем потоке с более теплой поверхностью водоема образуется туман, который распространяется над поверхностью водоема и на несколько километров в направлении горизонтальных масштабов. Возмущение метеорологических полей иллюстрирует рис.1 соответствующий варианту 5 в таблице 1 .

Возмущение удельной влажности воздуха, как показывает результаты численных экспериментов распространяется по ветру несколько километров.

Аналогичные данные для численного эксперимента с перегревом воды в $18^{\circ} \mathrm{C}$ по отношению к окружающему воздуху представлены на рис.2а,б,в (вариант 5). Как следовало ожидать, увеличение величины $\Delta \mathrm{T}$ приводят к более интенсивному и обширному туманообразованию. Граница тумана находится дальше от берега водохранилища по направлению ветра на несколько километров по сравнению с расчетами $\Delta \mathrm{T}=10^{\circ} \mathrm{C}$.

Кроме того, верхняя граница значительно высшее чем в эксперименте $\Delta \mathrm{T}=10^{\circ} \mathrm{C}$. Водность тумана на водоемом высшее почти 2 раза, чем первом эксперименте. Аналогичные результаты получены для температурных изменений и изменений влажности.

Надо отметить, при уменьшений размеров водоема вероятность образования тумана резко падает. Но и при отсутствии тумана водохранилищем возникают возмущение метеорологических элементов, которые также зависят от контраста температур натекающего воздуха и воды $\Delta \mathrm{T}$.

Разработанная методология использования численной нестационарной неоднородной модели пограничного слоя атмосферы позволяет возможность для оценки параметров адвективного в естественных условиях при наличии незамерзающих водоемов. Выявлены условия, способствующие образованию адвективного тумана и тумана парения в окресности незамерзающих водоемов. По мнению авторов, относительная простота модели позволяет осуществлять с ее помощью массовые расчеты изменения микроклимата в окрестности незамерзающих водоемов. 


\begin{tabular}{l|lrl|l|ll} 
& ISRA (India) & $=\mathbf{1 . 3 4 4}$ & SIS (USA) & $=\mathbf{0 . 9 1 2}$ & ICV (Poland) & $=\mathbf{6 . 6 3 0}$ \\
Impact Factor: & ISI (Dubai, UAE) $=\mathbf{0 . 8 2 9}$ & PUHL (Russia) $=\mathbf{0 . 2 3 4}$ & PIF (India) & $=\mathbf{1 . 9 4 0}$ \\
& GIF (Australia) & $\mathbf{0 . 5 6 4}$ & ESJI (KZ) & $=\mathbf{1 . 0 4 2}$ & IBI (India) & $=\mathbf{4 . 2 6 0}$ \\
& JIF & $\mathbf{1 . 5 0 0}$ & SJIF (Morocco) & $=\mathbf{2 . 0 3 1}$ & & \\
\hline
\end{tabular}

\section{References:}

1. Marchuk GI, Kondrat'ev KY, Kozoderov VV, Khvorst'yanov VI (1986) Oblaka i klimat. - L., Gidrometeoizdat, 1986.

2. Berlyand ME (1975) Sovremennye problemy atmosfernoy diffuzii i zagryazneniya atmosfery. - L., Gidrometeoizdat, 1975.

3. Marchuk GI (1982) Matematicheskoe modelirovanie $\mathrm{v}$ probleme okruzhayushchey sredy - M.: Nauka, 1982.

4. Yamada T (1979) An application of a threedimensional simplifited second - moment closure numerical model to atmospheric effects of large cooling pond. - Atmos. Environ., 1979, vol. 13.

5. Khvorst'yanov VI (1986) Modelirovanie i skhemy zon prosveta pri nazemnom rasseyanii pereokhlazhdennykh tumanov. - Meteorologiya i gidrologiya, 1986, №3.

6. Vager BG, Nadezhina ED (1979) Pogranichnyy sloy atmosfery $\mathrm{v}$ usloviyakh gorizontal'noy neodnorodnosti. - L., Gidrometeoizdat, 1979.

7. Orlanski IA (1976) simple boundary condition for unbounded hyperbolic tlows// Y.Comp.Phys.1976.V21.V3

8. Feygel'son EM (1970) Luchistyy teploobmen i oblaka. - L., Gidrometeoizdat, 1970.

9. Krasnokutskaya LD, Sushkevich TA (1977) Analiticheskoe predstavlenie integral'nykh funktsiy propuskaniya oblakov. - Izv.AN SSSR. Fizika atmosfery i okeana, 1977, t.13, № 5. 